\title{
Treatment of early non-response in patients with schizophrenia: assessing the efficacy of antipsychotic dose escalation
}

\author{
Antony Loebel ${ }^{1}$, Leslie Citrome $2,4^{*}$ (D) Christoph U. Correll ${ }^{3}$, Jane $\mathrm{Xu}^{1}$, Josephine Cucchiaro ${ }^{1}$ and John M. Kane ${ }^{3}$
}

\begin{abstract}
Background: Early non-response to antipsychotic treatment in patients with schizophrenia has been shown in multiple studies to predict poor response at short-term trial endpoint. Therefore, strategies to address the challenge of non-improvement early in the course of treatment are needed. A novel trial design was developed to assess the potential utility of antipsychotic dose escalation in patients with an inadequate initial treatment response. This design was embedded in a study intended to assess the efficacy of low dose lurasidone in patients with schizophrenia. The purpose of this report is to describe the background, rationale and design of this study that included a novel method for the assessment of the potential for dose-response in early non-responding patients with schizophrenia.
\end{abstract}

Methods/Design: In this 6-week, international, multicenter, double-blind trial, eligible adults with acute schizophrenia were randomized to receive fixed doses of lurasidone $20 \mathrm{mg} / \mathrm{day}, 80 \mathrm{mg} /$ day (active control), or placebo in a 1:2:1 ratio. Patients initially randomized to lurasidone $80 \mathrm{mg} /$ day who did not have a Positive and Negative Syndrome Scale total score improvement $\geq 20 \%$ at Week 2 were re-randomized on a $1: 1$ basis to receive either lurasidone $80 \mathrm{mg} /$ day or lurasidone $160 \mathrm{mg} /$ day for the remainder of the trial. All other groups remained on their initially assigned treatment. The formal primary objective of the study was to evaluate the efficacy of low-dose lurasidone (20 mg/day) compared to placebo; secondary objectives included evaluating the efficacy of lurasidone $80 \mathrm{mg} /$ day versus $160 \mathrm{mg} /$ day in early non-responders, and evaluating the efficacy of lurasidone in all subjects initially randomized to $80 \mathrm{mg} /$ day versus placebo.

Discussion: Since a lack of early improvement predicts poor response to short-term antipsychotic treatment in patients with schizophrenia, several treatment strategies have been proposed to enhance treatment outcome in early non-responders. A novel clinical trial design involving a placebo arm and re-randomization of early non-responders to increased or maintained antipsychotic dose was developed. The study design described in this report provides a robust method to assess the value of antipsychotic dose escalation in patients with schizophrenia who demonstrate poor initial treatment response.

Trial registration: ClinicalTrials.gov NCT01821378; initial registration March 22, 2013

Keywords: Antipsychotic, Dosing, Early responder, Lurasidone, Schizophrenia, Study design

\footnotetext{
* Correspondence: citrome@cnsconsultant.com

${ }^{2}$ New York Medical College, Valhalla, NY, USA

${ }^{4} 11$ Medical Park Drive, Suite 106, Pomona, NY 10970, USA

Full list of author information is available at the end of the article
}

(C) 2015 Loebel et al. Open Access This article is distributed under the terms of the Creative Commons Attribution 4.0 International License (http://creativecommons.org/licenses/by/4.0/), which permits unrestricted use, distribution, and reproduction in any medium, provided you give appropriate credit to the original author(s) and the source, provide a link to the Creative Commons license, and indicate if changes were made. The Creative Commons Public Domain Dedication waiver (http://creativecommons.org/publicdomain/zero/1.0/) applies to the data made available in this article, unless otherwise stated. 


\section{Background}

Treatment guidelines for patients with schizophrenia have typically suggested waiting 4 to 8 weeks to allow adequate time for a patient to respond to an antipsychotic drug prior to switching to another antipsychotic agent [1]. However, in a meta-analysis that included 7450 patients across 42 published studies, reductions in psychopathology were greater in Weeks one and two than in Weeks three and four, and this pattern was present even after the estimated effect of placebo treatment was removed and when results were restricted to the positive symptom subscales of the assessments used [2]. This finding has been replicated by subsequent investigations [3]. Lack of early response to antipsychotics in the acute treatment of schizophrenia has been shown in multiple studies to predict poor response at short-term trial endpoint [1, 4-6]. For example, among 131 patients with schizophrenia receiving open-label fluphenazine $20 \mathrm{mg} /$ day, every patient who experienced an improvement of less than $20 \%$ in Brief Psychiatric Rating Scale (BPRS) total score and $95 \%$ of patients who displayed a reduction of less than $20 \%$ in BPRS thought disturbance factor score following 1 week of treatment were classified as non-responders after 4 weeks of treatment [4]. A pooled analysis was conducted of five randomized, double-blind clinical trials that compared olanzapine to other second-generation antipsychotics in patients with schizophrenia and related disorders. Early response was defined as $\geq 20 \%$ improvement on the Positive and Negative Syndrome Scale (PANSS) total score at 2 weeks. Conditional probabilities (sensitivity, specificity, positive and negative predictive values) were used to characterize the likelihood of "subsequent response" to treatment (i.e., $\geq 40 \%$ improvement on the PANSS total score with treatment up to 3 months) [1]. For the receiver operating characteristics curve, the area under the curve was at least 0.75 for all criteria used to assess subsequent response, indicating that the magnitude of early symptom improvement at 2 weeks could predict subsequent response at 3 months. $80 \%$ of nonresponders at endpoint were correctly identified as early non-responders at 2 weeks (specificity) and $84 \%$ of early non-responders were non-responders at endpoint (negative predictive value). However, compared to early nonresponse, early response was not as strong a predictor of subsequent response.

When early non-response occurs, potential patient management strategies include increasing the dose of the currently administered antipsychotic medication within the approved dose range or beyond, adding adjunctive medications or switching to another antipsychotic [7]. Another approach to early non-response is to simply wait and continue the initial treatment. However, the chances of achieving a robust antipsychotic response at the starting dose are low in the face of early non-response [1].

Switching to another antipsychotic in the face of early non-response has not been shown to yield substantially better outcomes. In a randomized double-blind, flexibledosed, 12-week study in patients diagnosed with schizophrenia or schizoaffective disorder, early non-response to risperidone was used to study the potential benefit of switching from risperidone to olanzapine [5]. Early nonresponse was defined as failure to achieve a $\geq 20 \%$ improvement on the PANSS total score following 2 weeks of risperidone treatment. Early non-responders were randomized to continue on risperidone $2-6 \mathrm{mg} /$ day or switch to olanzapine $10-20 \mathrm{mg} /$ day for ten additional weeks (early responders remained on risperidone). Early response/non-response was highly predictive of subsequent clinical outcomes. Switching from risperidone in early non-responders to olanzapine at week two resulted in a small, but significantly greater reduction in PANSS total score $(-16$ points versus -12 points for those staying on risperidone). Of note, the reduction in PANSS total score following the switch to olanzapine was greater among those patients who were still moderately ill at 2 weeks $(-22$ points versus -16 points for those staying on risperidone) [5]. A robust decrease in the PANSS total score observed amongst early responders to risperidone ( -40 points) reinforces the clinical observation that early responders and early non-responders may comprise two distinct patient populations.

Dose escalation in the presence of early non-response has not been well studied. Both switching and dose escalation were examined in an early study in acute schizophrenia where patients received open-label fluphenazine $20 \mathrm{mg} /$ day for 4 weeks [8]. Subjects failing to meet response criteria, which were very stringent (mild or better on each of the four BPRS psychotic items and a rating of much improved or better on the Clinical Global Impressions scale global improvement item) after 4 weeks were then randomized, double-blind, to continue fluphenazine $20 \mathrm{mg} /$ day, dose escalation to fluphenazine $80 \mathrm{mg} /$ day, or switch to haloperidol $20 \mathrm{mg} /$ day [8]. No differences in efficacy were found among these treatment strategies. Dose-escalation in the presence of inadequate response has also been examined in patients with schizophrenia receiving quetiapine $[9,10]$ and ziprasidone [11]. In these studies, subjects were required to have demonstrated failure on a therapeutic dose in order to be eligible to be randomized to either continue their original dose or to receive a supratherapeutic dose (quetiapine $1200 \mathrm{mg}$ /day or ziprasidone $320 \mathrm{mg} /$ day depending on the study) under double-blind conditions. In these three studies the higher dose was not superior to the lower. However, none of these four studies used a 2-week decision time point for 
randomization to dose escalation vs. standard, using instead $\geq 4$ weeks [8-10] or $\geq 3$ weeks [11]. One study [9] used less than minimal improvement $(\leq 15 \%$ reduction on PANSS total sore) as an "early" non-response definition. In addition, these studies used a variety of criteria to assess non-response, some of which were imprecise, such as having at least one moderate positive symptom [8] a relatively high threshold of $<30 \%$ PANSS total improvement [10], or "remaining symptomatic" [11]. Moreover, none of these studies included a placebo arm, which may have created a significant expectation bias towards improvement of all subjects.

Lurasidone is a second-generation antipsychotic agent that has received regulatory approval for the treatment of adults with schizophrenia and bipolar depression (both as monotherapy and as adjunct to lithium or valproate) in the US and other countries [12, 13]. The recommended dose range for lurasidone for the treatment of schizophrenia is $40-160 \mathrm{mg} /$ day. There is evidence suggesting a dose response, with lurasidone $160 \mathrm{mg} /$ day resulting in more robust reductions in psychopathology than $80 \mathrm{mg} /$ day in one 6-week acute study [14]. In a post hoc analysis of pooled data from five, 6-week, acute studies of lurasidone, least square mean change-frombaseline in PANSS exhibited a linear trend relative to dose of lurasidone [15]. However, there is a clinical need to understand optimal dosing strategies for lurasidone, including whether dose escalation may be effective in patients with little or no early improvement. As part of the postmarketing commitments made to the US Food and Drug Administration, the utilization of lurasidone in the treatment of adults with schizophrenia with a dose lower than $40 \mathrm{mg}$ (e.g., $20 \mathrm{mg}$ daily) was to be studied through an adequate and well-controlled trial [16]. This report describes the methodology of a novel clinical trial design that in addition to examining the efficacy of lurasidone $20 \mathrm{mg} /$ day, assessed the efficacy and safety of dose escalation in patients with early non-response by re-randomizing early non-responders to either continue $80 \mathrm{mg} /$ day (the initially assigned dose) or to receive $160 \mathrm{mg} /$ day. (Trial Registration: ClinicalTrials.gov NCT01821378).

\section{Methods/Design}

The study schematic is illustrated in Fig. 1. In this 6week, international, multi-center, double-blind trial, eligible patients were randomized (via a centralized interactive voice/web response system) to receive fixed doses of lurasidone $20 \mathrm{mg} /$ day, $80 \mathrm{mg} /$ day (active control arm), or placebo on a 1:2:1 ratio. At 2 weeks all subjects were evaluated for early non-response and patients were re-randomized according to the following scheme: subjects assigned to lurasidone $20 \mathrm{mg} /$ day or to placebo continued to receive the same intervention; patients previously randomized to lurasidone $80 \mathrm{mg} /$ day and who had a PANSS improvement $\geq 20 \%$ at Week two remained on lurasidone $80 \mathrm{mg} /$ day; patients previously randomized to lurasidone $80 \mathrm{mg} /$ day and who had a PANSS improvement $<20 \%$ at Week two were randomized on a $1: 1$ basis to receive either lurasidone $80 \mathrm{mg} /$ day or lurasidone $160 \mathrm{mg} /$ day for the remainder of the trial.

The study was conducted between May 2013 and June 2014 at 64 sites in the United States, Russia, Romania, Ukraine, Slovakia, and Colombia. The study is undergoing the final data analysis stage.

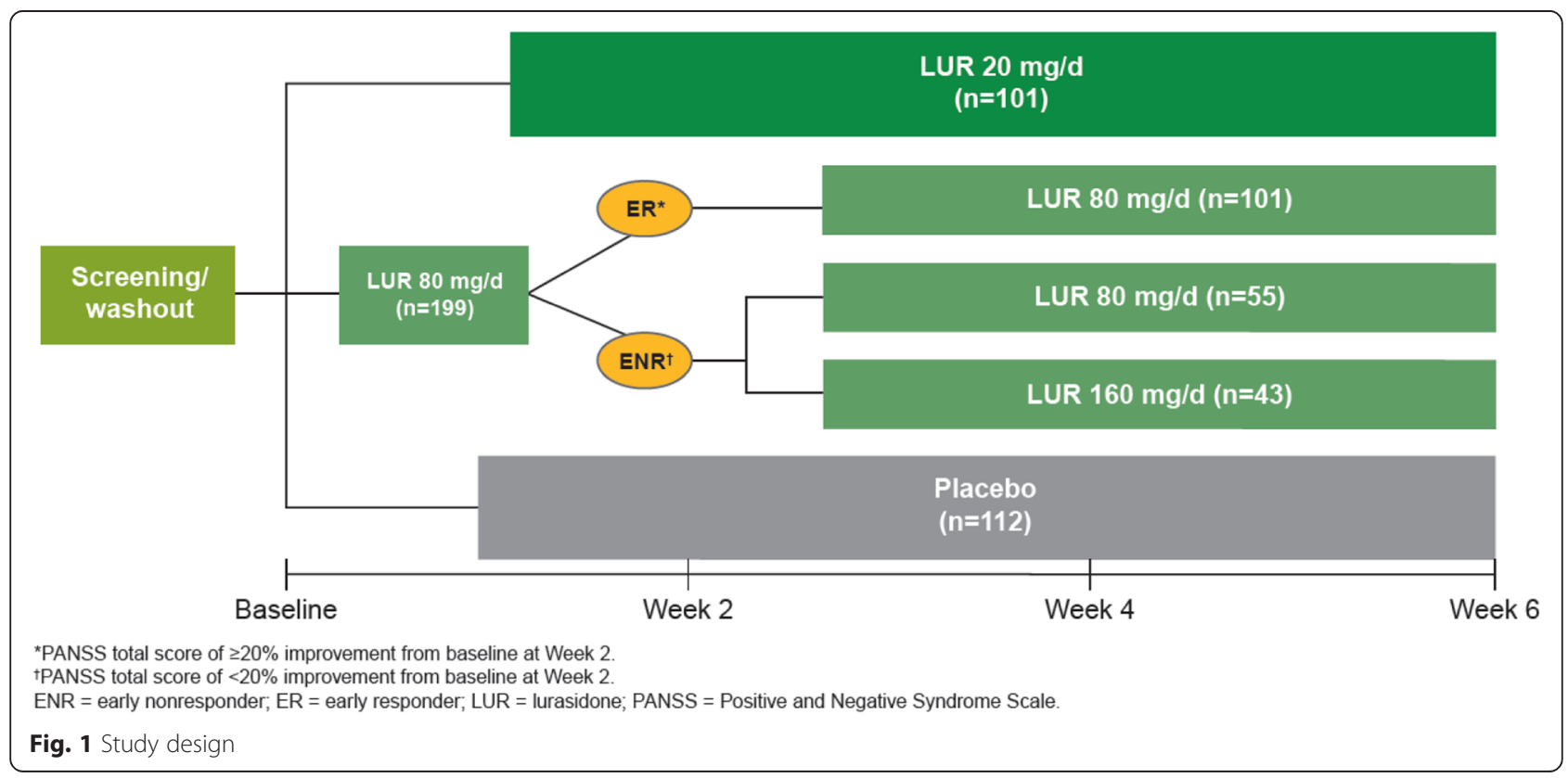




\section{Objectives}

The formal primary objective of the study was to evaluate the efficacy of low-dose lurasidone $(20 \mathrm{mg} /$ day $)$ compared to placebo in patients with an acute exacerbation of schizophrenia; secondary objectives included evaluating the efficacy of lurasidone $80 \mathrm{mg} /$ day versus $160 \mathrm{mg} /$ day in early non-responders, and evaluating the efficacy of lurasidone in all subjects initially randomized to $80 \mathrm{mg} /$ day versus placebo.

\section{Eligibility criteria}

Eligible for participation were men and women between the ages of $18-75$ years inclusive, with a diagnosis of DSM-IV-TR schizophrenia, duration of illness $\geq$ 6 months, PANSS total score $\geq 80$ and a PANSS subscale score $\geq 4$ (moderate) on two or more of the following PANSS items: delusions, conceptual disorganization, hallucinations, and unusual thought content at screening and baseline, Clinical Global Impressions-Severity of Illness (CGI-S) score of $\geq 4$ (moderately ill) at screening and baseline, presence of an acute exacerbation of psychotic symptoms (no longer than 2 months) and marked deterioration of function from baseline (by history) or hospitalized for the purpose of treating an acute psychotic exacerbation for two consecutive weeks or less immediately before screening, and a stable living arrangement. Subjects were also required to be medically stable and those with acute or significant medical conditions were excluded. Subjects who were on stable medication regiments including oral hypoglycemic agents, antihypertensive agents, or thyroid hormone replacement were eligible to participate. Concomitant medications that are CYP3A4 inducers and inhibitors were not permitted. Antidepressants and mood stabilizers (e.g., lithium, divalproex/valproic acid, carbamazepine, etc.) were not permitted. Subjects with treatment resistance to antipsychotic therapy were excluded. The study was approved by an independent ethics committee/institutional review board associated with each investigational site and was conducted in accordance with the International Conference on Harmonisation Good Clinical Practices guidelines and with the ethical principles of the Declaration of Helsinki. A separate file is available with a list of all the names of the independent ethics committees/institutional review boards (Additional file 1). All study participants reviewed and signed an informed consent document explaining study procedures and potential risks before study entry.

\section{Outcome measures}

The PANSS and CGI-S were obtained at screening, baseline (Day 1), and at Days 4, 8, 15, 22, 29, 36 and 43. Outcomes measured included change from baseline in PANSS total score at Week six for each of the lurasidone groups versus placebo. Secondary outcome measures included change in the CGI-S score, PANSS subscale scores, the Montgomery-Asberg Depression Rating Scale (MADRS) total score, and the proportion of subjects who achieved a response, defined as $\geq 20 \%$ improvement from baseline in PANSS total score. Other measures included adverse events (AEs), AEs leading to discontinuations, and serious AEs, physical examination and laboratory measures, and assessments of extrapyramidal symptoms, suicidality, general health status, functioning, adherence, medication satisfaction, and drop-out risk.

\section{Study flow}

Subjects who met entry criteria (after a 14 day screening phase) entered a 3- to 7-day washout period and remained hospitalized for the duration of the washout. Subjects who demonstrated a decrease (improvement) of $\geq 20 \%$ in the PANSS score between screening and baseline visits or whose PANSS total score fell below 80 at baseline were excluded from this efficacy study. Study medication was administered once daily in the evening with a meal (e.g., dinner) of at least 350 calories or within $30 \mathrm{~min}$ of eating. Hospitalization was mandatory through Week three, after which subjects were eligible for hospital discharge if they met specific clinical stability and discharge criteria and could be followed as outpatients for the remainder of the study. Subjects who completed the study and those who discontinued the study early had a follow-up visit 7 days $( \pm 2)$ after the last dose of study medication.

\section{Data analysis}

The study sample size was projected to be 100 subjects in the placebo group, 100 subjects in the lurasidone $20 \mathrm{mg}$ group, and 200 subjects in the lurasidone $80 \mathrm{mg}$ group as determined by two-sample t-tests using nQuery advisor (Version 7.0), based on the treatment effect sizes observed in previous lurasidone studies, to provide a power of $80 \%$ to discern differences between lurasidone $20 \mathrm{mg} /$ day and placebo, as well as between lurasidone $80 \mathrm{mg} /$ day and lurasidone $160 \mathrm{mg}$ /day among the early non-responders, after accounting for potential early drop-outs.

The pre-planned primary efficacy analysis was the examination of the change from baseline in PANSS total score at Week six for lurasidone $20 \mathrm{mg} / \mathrm{d}$ versus placebo using a mixed model for repeated measures (MMRM) for the Intent-to-Treat (ITT) population (defined as subjects who were randomized at baseline, received at least one dose of study drug, and had both baseline and at least one post-baseline assessment of the efficacy measure). The MMRM model included treatment, visit, pooled center, baseline scores, and a treatment-by-visit interaction term, using an unstructured covariance 
matrix. For the key secondary efficacy analysis, the change from baseline to Week 6 in CGI-S score for lurasidone $20 \mathrm{mg}$ versus placebo was analyzed for the ITT population using a similar MMRM model.

The early non-responder ITT population was defined as all subjects who were randomized at Week two, received at least one dose of study medication after the randomization at Week two, and had both a Week two efficacy assessment and at least one efficacy assessment after Week two. Analysis of key secondary efficacy outcomes, change from baseline in PANSS (total score and subscale scores) and CGI-S score for the 80-160 mg early non-responder lurasidone groups was analyzed using MMRM. Change from baseline in MADRS total score was analyzed using analysis of covariance using both observed case and last observation carried forward approaches.

Analyses for PANSS responders were performed using a logistic regression model, which included baseline PANSS total score, pooled center, and treatment.

The safety analyses were conducted using the Safety population (defined as subjects who were randomized and received at least one dose of study medication).

\section{Discussion}

We propose that the study design characteristics reported here provide a robust method to assess the potential for dose-response in early non-responding patients with schizophrenia (or other psychiatric illness).

The key design elements incorporated into this study were: a) initial randomization of patients in double-blind fashion to lurasidone and placebo groups; b) prospective assessment of non-response to study treatment; d) selection of the week two visit to assess level of response to study treatment; e) clear, operationalized definition of non-response; f) re-randomization of early nonresponders treated with lurasidone to high dose or standard dose treatment in double-blind fashion and g) assessment of response to lurasidone dose escalation vs continuation of standard dose at the week 6 study visit (4 weeks post-randomization of early non-responders). To our knowledge, the study protocol reported here represents the first study ever conducted that includes these design elements.

Initial lack of improvement is an important predictor of short-term treatment outcome across various psychiatric disorders. This finding has been reported not only for schizophrenia [1], but also in the treatment of major depressive disorder $[17,18]$ and acute bipolar mania [19]. Several treatment strategies have been proposed to enhance outcome in patients who are early nonresponders [7], but few have been rigorously tested.

Using the early antipsychotic response/non-response paradigm, proposed clinical trial designs include an "early responder randomized discontinuation design" where all patients are assigned to the active drug, and only those who had at least a minimal response at 2 weeks are enrolled in a double-blind, placebocontrolled discontinuation trial, enriching the placebo controlled trial portion with true drug responders [20]. In the mirror image "early non-responder randomized dose increase or augmentation design," early nonresponders at 2 weeks are assigned to staying on the medication or going either to a higher dose or an augmentation agent [20]. Our study incorporated the latter methodology as a way of exploring questions regarding dose response in early non-responders to antipsychotic treatment. Importantly, rather than addressing whether or not higher doses of a specific antipsychotic are more efficacious for "all-comers" [21], or in patients not showing clinical response after an adequate trial of 3 weeks or longer [8-11] (using various definitions of nonresponse that allowed for more than minimal improvement except in one study [9]), this protocol tested whether dose escalation was effective in patients with a clearly inadequate initial treatment response at week two. The population being examined was thus enriched by the specific selection of subjects who prospectively failed to achieve at least minimal response in the first two weeks of the trial. This design permitted a clear demonstration of dose-response in early non-improvers, which may not be evident in studies involving "all comers" or in studies with variable time to early response criteria and imprecise early response definitions. Moreover, previous studies of dose escalation in the face of inadequate response have been confounded by expectation bias that may be present in the absence of a placebo control. The current study minimized this effect by the inclusion of a placebo arm. Whether dose escalation in patients with schizophrenia is an efficacious strategy has important implications regarding optimizing outcomes, maximizing the opportunity for recovery, and managing costs.

The selection of Week two as the point in time to determine non-response was based on prior published work in this area $[1-3,5]$. This time point is further supported by naturalistic studies, enhancing the generalizability of this concept [22].

Limitations to the trial included the lack of intermediate doses of lurasidone (i.e., $120 \mathrm{mg} /$ day) to be tested as part of the early non-responder design either as starting or target dose, and that the trial length was only 6 weeks; longer-term outcomes were not tested in this design. Moreover, doses of lurasidone that exceed the maximum recommended amount of $160 \mathrm{mg} /$ day were not tested. A 24-week double-blind randomized controlled trial comparing lurasidone $240 \mathrm{mg} /$ day versus $80 \mathrm{mg} /$ day in treatment resistant schizophrenia or schizoaffective disorder 
is in progress (NCT01569659). Generalizability of the results of dose escalation of lurasidone in this study to other antipsychotics is not known. SPIRIT checklist is available in a separate file (Additional file 2).

\section{Summary}

The clinical management of early non-response to antipsychotic medication treatment is a common clinical conundrum. This protocol reported here was designed in part to explore the strategy of antipsychotic dose escalation in the presence of early non-response to standard dose treatment. The study design reflected the early non-response paradigm and included blinded rerandomization of early non-responders, as well as an effort to minimize expectation bias and enhance signal detection by the inclusion of a placebo control. In this context, this is the first rigorous study to randomize well-defined, early non-responding patients with schizophrenia to either maintain or escalate an initial dose of antipsychotic treatment in a double-blind fashion, incorporating a placebo-control group.

\section{Additional files}

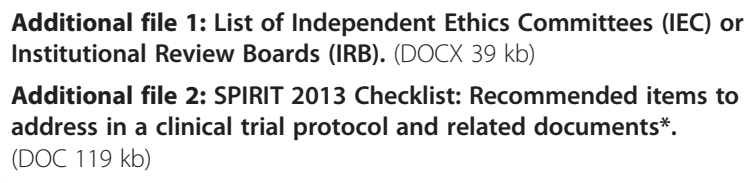

\section{Abbreviations}

AE: Adverse event; BPRS: Brief Psychiatric Rating Scale; CGI-S: Clinical Global Impressions-Severity of IIIness; DSM-IV-TR: Diagnostic and Statistical Manual of Mental Disorders, Fourth Edition, Text Revision; ITT: Intent-to-Treat; MADRS: Montgomery-Asberg Depression Rating Scale; MMRM: mixed model for repeated measures; PANSS: Positive and Negative Syndrome Scale.

\section{Competing interests}

A. Loebel, J. Xu, and J. Cucchiaro are full-time employees of Sunovion Pharmaceuticals. In the past 12 months, L. Citrome, was a consultant for, has received honoraria from, or has conducted clinical research supported by the following: Alkermes, AstraZeneca, Bristol-Myers Squibb, Eli Lilly, Forest, Genentech, Janssen, Merck, Mylan, Novartis, Noven, Otsuka, Pfizer, Shire, and Sunovion. C.U. Correll was a consultant for, has received honoraria from, or has conducted clinical research supported by the following: AbbVie, Alkermes, Bristol-Myers Squibb, Eli Lilly, Genentech, Gerson Lehrman Group, IntraCellular Therapies, Janssen/J\&J, Lundbeck, MedAvante, Medscape, Otsuka, Pfizer, ProPhase, Reviva, Roche, Sunovion, Supernus, and Takeda. J.M. Kane was a consultant for, has received honoraria from, or has conducted clinical research supported by the following: Alkermes, Bristol-Myers Squibb, Eli Lilly, Forrest Labs, Genentech, Intracellular Therapies, Janssen, Johnson and Johnson, Lundbeck, Merck, Novartis, Otsuka, Pfizer, Reviva, Roche, Sunovion, Takeda. He is a shareholder of MedAvante.

\section{Authors' contributions}

AL, JX and JC developed the protocol. LC created the first draft of this manuscript. AL, CUC, JX, JC, and JMK provided comments, revisions, and additional text. All authors (AL, LC, CUC, JX, JC, and JMK) read and approved the final manuscript.

\section{Acknowledgements}

This work was supported by Sunovion Pharmaceuticals Inc., Marlborough, MA and Fort Lee, NJ.

\section{Author details}

${ }^{1}$ Sunovion Pharmaceuticals Inc., Fort Lee, NJ, USA. ${ }^{2}$ New York Medical College, Valhalla, NY, USA. ${ }^{3}$ The Zucker Hillside Hospital, Glen Oaks, and the Hofstra North Shore-LIJ School of Medicine, Hempstead, NY, USA. ${ }^{4} 11$ Medical Park Drive, Suite 106, Pomona, NY 10970, USA.

Received: 17 June 2015 Accepted: 2 October 2015

Published online: 31 October 2015

\section{References}

1. Kinon BJ, Chen L, Ascher-Svanum H, Stauffer VL, Kollack-Walker S, Sniadecki $J L$, et al. Predicting response to atypical antipsychotics based on early response in the treatment of schizophrenia. Schizophr Res. 2008;102:230-40.

2. Agid O, Kapur S, Arenovich T, Zipursky RB. Delayed-onset hypothesis of antipsychotic action: a hypothesis tested and rejected. Arch Gen Psychiatry. 2003;60:1228-35.

3. Leucht S, Busch R, Hamann J, Kissling W, Kane JM. Early-onset hypothesis of antipsychotic drug action: a hypothesis tested, confirmed and extended. Biol Psychiatry. 2005;57:1543-9.

4. Correll CU, Malhotra AK, Kaushik S, McMeniman M, Kane JM. Early prediction of antipsychotic response in schizophrenia. Am J Psychiatry. 2013;160:2063-5.

5. Kinon BJ, Chen L, Ascher-Svanum H, Stauffer VL, Kollack-Walker S, Zhou W, et al. Early response to antipsychotic drug therapy as a clinical marker of subsequent response in the treatment of schizophrenia. Neuropsychopharmacology. 2010;35:581-90.

6. Correll CU, Pikalov A, Hsu J, Cucchiaro J, Goldman R, Loebel A. Early improvement predicts endpoint response to lurasidone in schizophrenia: pooled analysis of five double-blind trials. Poster presentation. American Psychiatric Association, May 3-7, 2014, New York, NY

7. Hatta K, Ito H. Strategies for Early Non-response to Antipsychotic Drugs in the Treatment of Acute-phase Schizophrenia. Clin Psychopharmacol Neurosci. 2014;12:1-7.

8. Kinon BJ, Kane JM, Johns C, Perovich R, Ismi M, Koreen A, et al. Treatment of neuroleptic-resistant schizophrenic relapse. Psychopharmacol Bull. 1993;29:309-14.

9. Lindenmayer JP, Citrome L, Khan A, Kaushik S, Kaushik S. A randomized, double-blind, parallel-group, fixed-dose, clinical trial of quetiapine at 600 versus $1200 \mathrm{mg} / \mathrm{d}$ for patients with treatment-resistant schizophrenia or schizoaffective disorder. J Clin Psychopharmacol. 2011;31:160-8.

10. Honer WG, MacEwan GW, Gendron A, Stip E, Labelle A, Williams R, et al. A randomized, double-blind, placebo-controlled study of the safety and tolerability of high-dose quetiapine in patients with persistent symptoms of schizophrenia or schizoaffective disorder. J Clin Psychiatry. 2012;73:13-20.

11. Goff DC, McEvoy JP, Citrome L, Mech AW, Bustillo JR, Gil R, et al. High-dose oral ziprasidone versus conventional dosing in schizophrenia patients with residual symptoms: the ZEBRAS study. J Clin Psychopharmacol. 2013;33:485-90.

12. Latuda: US Package Insert. 2013. [http://www.latuda.com/ LatudaPrescribingInformation.pdf]. Accessed October 12, 2015

13. Latuda: EU Summary of Product Characteristics. 2014. [http:// www.ema.europa.eu/docs/en_GB/document_library/EPAR_-_Product_ Information/human/002713/WC500164683.pdf]. Accessed October 12, 2015.

14. Loebel A, Cucchiaro J, Sarma K, Xu L, Hsu C, Kalali AH, et al. Efficacy and safety of lurasidone $80 \mathrm{mg} /$ day and $160 \mathrm{mg} /$ day in the treatment of schizophrenia: a randomized, double-blind, placebo- and active-controlled trial. Schizophr Res. 2013;145:101-9.

15. Chapel S, Chiu YY, Hsu J, Xu J, Cucchiaro, Loebel A. Dose-response model of lurasidone treatment in schizophrenia. Poster presentation. American Psychiatric Association, May 5-9, 2012, Philadelphia, PA

16. US Food and Drug Administration: NDA Approval letter. [http:// www.accessdata.fda.gov/drugsatfda_docs/appletter/2010/200603s000ltr.pdf]. Accessed October 12, 2015.

17. Vermeiden $M$, Kamperman $A M$, Vulink $M E$, van den Broek $W W$, Birkenhäger TK. Early improvement as a predictor of eventual antidepressant treatment response in severely depressed inpatients. Psychopharmacology (Berl). 2015;232:1347-56. 
18. Szegedi A, Jansen WT, van Willigenburg AP, van der Meulen E, Stassen HH, Thase ME. Early improvement in the first 2 weeks as a predictor of treatment outcome in patients with major depressive disorder: a meta-analysis including 6562 patients. J Clin Psychiatry. 2009;70:344-53.

19. Kemp DE, Johnson E, Wang WV, Tohen M, Calabrese JR. Clinical utility of early improvement to predict response or remission in acute mania: focus on olanzapine and risperidone. J Clin Psychiatry. 2011;72:1236-41.

20. Correll CU, Kishimoto T, Kane JM. Randomized controlled trials in schizophrenia: opportunities, limitations, and trial design alternatives. Dialogues Clin Neurosci. 2011;13:155-72.

21. Kinon BJ, Volavka J, Stauffer V, Edwards SE, Liu-Seifert H, Chen L, et al. Standard and higher dose of olanzapine in patients with schizophrenia or schizoaffective disorder: a randomized, double-blind, fixed-dose study. J Clin Psychopharmacol. 2008;28:392-400.

22. Jäger $M$, Schmauss $M$, Laux G, Pfeiffer $H$, Naber D, Schmidt LG, et al. Early improvement as a predictor of remission and response in schizophrenia: Results from a naturalistic study. Eur Psychiatry. 2009;24:501-6.

\section{Submit your next manuscript to BioMed Central and take full advantage of:}

- Convenient online submission

- Thorough peer review

- No space constraints or color figure charges

- Immediate publication on acceptance

- Inclusion in PubMed, CAS, Scopus and Google Scholar

- Research which is freely available for redistribution 